\title{
openheart Re-engineering the post-myocardial infarction medicines optimisation pathway: a retrospective analysis of a joint consultant pharmacist and cardiologist clinic model
}

\author{
Rani Khatib, ${ }^{1,2,3}$ Nasrin Patel, ${ }^{1,2}$ Una Laverty, ${ }^{1}$ Gina Mcgawley, ${ }^{2}$ Jim McLenachan, ${ }^{2}$ \\ Sarah Shield, ${ }^{4}$ Alistair S Hall ${ }^{2,3}$
}

To cite: Khatib R, Patel N, Laverty U, et al. Re-engineering the post-myocardial infarction medicines optimisation pathway: a retrospective analysis of a joint consultant pharmacist and cardiologist clinic model. Open Heart 2018;5:e000921. doi:10.1136/ openhrt-2018-000921

Received 15 August 2018 Revised 5 November 2018 Accepted 15 November 2018

Check for updates

(c) Author(s) (or their employer(s)) 2018. Re-use permitted under CC BY-NC. No commercial re-use. See rights and permissions. Published by BMJ.

${ }^{1}$ Medicines Management \& Pharmacy Services, Leeds Teaching Hospital NHS Trust, Leeds, UK

${ }^{2}$ Cardiology Department, Leeds Teaching Hospitals NHS Trust, Leeds, UK

${ }^{3}$ Leeds Institute of

Cardiovascular and Metabolic Medicine, Universityof Leeds, Leeds, UK

${ }^{4}$ NHS Relations, AstraZeneca, Horizon Place, Capability Green, Luton, UK

\section{Correspondence to}

Dr Rani Khatib; r.khatib@leeds. ac.uk

\section{ABSTRACT}

Background Inadequate medicines optimisation and adherence are significant problems among patients taking secondary prevention medications following myocardial infarction (MI). A novel joint consultant cardiology pharmacist and cardiologist medicines optimisation clinic was initiated for patients recently discharged following Ml. Methods Patients completed a locally developed tool, the 'My Experience of Taking Medicines' questionnaire, designed to allow sharing of barriers to adherence with medications. They then attended a clinic with the consultant pharmacist or cardiologist (or both). Secondary prevention medicines needs and barriers to adherence were identified and discussed, and an action plan developed. The data provided are from a retrospective review of 270 post-Ml patients attending the service between October 2015 and December 2016.

Results Mean age was 67.3 years and $67.8 \%$ were male. The mean time from discharge to first outpatient clinic attendance was reduced by $56.1 \%$ ( 49.4 days vs 88 days before the service began). More than $95 \%$ of patient without planned non-pharmacological intervention postdischarge did not need a cardiologist's input. Levels of medicines optimisation were improved substantially after attendance: patients receiving a recommended angiotensin-converting enzyme inhibitor/angiotensin receptor blocker dose increased from $16.3 \%$ to $73.9 \%$ $(p<0.001)$; patients receiving a recommended betablocker dose increased from $6.2 \%$ to $46.1 \%$ ( $p<0.001$ ). Patient concerns about their medications were significantly decreased (all $p<0.001$ ). Rates of non-adherence fell by $42.6 \%-70.8 \%$ at $3-6$ months post-clinic. Readmission rates also declined after the service opened.

Conclusions A medicines optimisation and patient adherence strategy based on a joint consultant cardiology pharmacist and cardiologist clinic can improve both adherence and outcomes post-Ml.

\section{INTRODUCTION}

In patients who have experienced a myocardial infarction (MI), it is essential to initiate a long-term programme of secondary
Key questions

What is already known about this subject?

- The benefits of secondary prevention medicines (SPM) post-myocardial infarction (MI) are substantial and well established. However, there is plenty of evidence to show that many opportunities are missed by not prescribing these medicines at optimal doses and/or by lack of adherence to these medicines. It is estimated that up to $50 \%$ of patients do not take at least one of their SPM as prescribed. Interventions to improve adherence cannot be one size fit all, they need to be tailored to patient's needs.

What does this study add?

- The study introduces a new model of working which involves the support of a cardiology consultant pharmacist and a review of patients' medicines needs post-Ml. It offers the patients the opportunity to identify any actual and potential barriers to SPM adherence to be addressed in the first outpatient cardiology clinic. The new model improved levels of optimisation of SPM, addressed concerns about SPM, reduced self-reported non-adherence, acute coronary syndrome readmissions (compared to before service introduced) and improved persistence with guidelines recommended SPM at 12 months postdischarge.

How might this impact on clinical practice?

- The new model shows the need to re-examine how outpatient follow up is arranged post-MI in terms of patient's needs, healthcare professionals involved and adopting a new approach based on the principles of medicines optimisation and adherence to deliver a more patient-centred medicines review. This is likely to improve outcomes and reduce many missed opportunities post-MI.

prevention through risk factor modification. ${ }^{1-3}$ Optimisation of drug therapies is a key element of this, ensuring that patients 
are receiving all of the required classes of treatment at an appropriate dose.

However, in a prospective, observational study conducted at a centre in the UK, more than $25 \%$ of patients with pre-existing coronary heart disease who were readmitted with an acute coronary syndrome were receiving a suboptimal secondary prevention drug regimen. ${ }^{4}$ Many of these patients were not achieving cholesterol and blood pressure targets, which could have been a major contributor to their re-presentation to hospital.

Furthermore, even among patients who are prescribed an optimal secondary prevention regimen, non-adherence can be a significant problem. Up to half of all medicines prescribed for long-term conditions may not be taken as recommended, with significant potential implications for the patient, healthcare system and society as a whole..$^{5}$ A meta-analysis of data from almost 400000 patients from 20 studies assessing adherence to drugs that prevent cardiovascular disease estimated that adherence was around $57 \%$ after a median of 24 months. ${ }^{6}$ The impact on outcomes can be substantial. For example, among patients with elevated cholesterol prescribed a statin, non-adherence was associated with a $26 \%$ increase in cardiovascular-related hospitalisations compared with adherent patients. ${ }^{7}$ In a recent meta-analysis, the relative risks of all-cause mortality in patients with good versus poor $(<80 \%)$, adherence were 0.55 and 0.71 in those taking statins and antihypertensive agents, respectively. ${ }^{8}$ More specifically in secondary prevention, adherence with medications post-MI has been shown to decrease the likelihood of readmission for a cardiovascular-related issue. $^{8}$

Local data from 500 patients with coronary artery disease in the West Yorkshire area of the UK found that $44 \%$ were not adherent to at least one secondary prevention medicine. ${ }^{9}$ To try to address this issue, a novel joint consultant cardiology pharmacist and cardiologist medicines optimisation clinic was initiated for patients who have recently been discharged following MI. The aims were to ensure that patients receive a comprehensive review of their secondary prevention medicine needs; to allow patients to share their medicines-taking experience and receive the necessary support to maximise benefit and minimise the risk of adverse drug reactions and, ultimately, to improve patient outcomes.

\section{METHODS}

\section{Service design}

The post-MI medicines optimisation multidisciplinary team (MDT) clinic was initiated in October 2015, as a collaboration between the Cardiology and Pharmacy Departments of Leeds Teaching Hospitals National Health Service Trust. Initially, it operated as a pilot programme, but the service development project was later continued on a larger scale as part of a joint working agreement between Leeds Teaching Hospitals and AstraZeneca.
Consecutive patients from the city of Leeds, UK, who were admitted to Cardiology with MI were triaged to attend a post-discharge follow-up clinic in the new service. In advance of that visit, patients were sent a locally developed tool known as the 'My Experience of Taking Medicines' (MYMEDS) questionnaire. This tool was specifically designed to enable patients to share any actual or potential barriers that they may have with adherence to secondary prevention medicines.

Patients then attended the post-MI medicines optimisation MDT clinic. Patients who did not need any further non-pharmacological interventions saw the consultant cardiology pharmacist with the option of a review by a cardiologist if needed. Patients who did need further interventions (eg, staged percutaneous coronary intervention) saw a cardiologist, with the option of referral to the consultant pharmacist. Support was provided by a cardiology research nurse to collect data.

Most patients attended the 'Standard Medicines Optimisation Clinic', typically lasting 20-25 min; however, post-MI patients with complex medicines-related issues (eg, documented or high risk for poor adherence or intolerance to any secondary prevention medicines) attended an 'Advanced Medicines Optimisation Clinic', which included a longer consultation (around $45 \mathrm{~min}$ ), allowing time to conduct a thorough review.

Both types of consultations took a patient-centred approach, striving first to meet the needs of the individual concerned. Secondary prevention medicines and barriers to adherence were identified and discussed based on MYMEDS.

Following on from the consultation, patients were provided with a summary of the discussions and a full management plan, including the main action points. To ensure continuity of care, this was also shared with their general practitioner (GP) and with the cardiac rehabilitation team.

The present analysis is a retrospective review of data from 270 post-MI patients attending the service between October 2015 and December 2016. It was conducted in accordance with the World Medical Association Declaration of Helsinki. As this was a service development project, in line with local policy, no Ethics Committee approval was needed. However, the project had a steering committee that monitored the project and tracked its progress on a quarterly basis. The committee included the clinical directors of cardiology and pharmacy, cardiologists, pharmacists, a cardiology nurse, a GP and an AstraZeneca representative and had input from a patient representative.

\section{Assessments}

Patients completed the My Experience of Taking Medicine (MYMEDS) questionnaire before attending the clinic and brought it with them for discussion during the consultation.

Within the MYMEDS questionnaire, patient listed the medicines that they were taking and these were assessed 
against national and international guideline recommendations. Patients were asked about their understanding of why these medicines were prescribed, any concerns they had about them and any practical barriers to taking them and were also asked to report whether they took each of their medicines as recommended by the prescriber. The answers were used to estimate levels of self-reported non-adherence. Optimisation of the doses of secondary prevention medicines (ACE inhibitors, angiotensin receptor blockers (ARBs), beta blockers, statins, antiplatelet agents and eplerenone where indicated) was considered during the clinic. Further review was made of other antianginal drugs and the need for gastroprotective drugs, if required.

After attendance, patients were also asked to rate their experience of the clinic using an anonymous feedback questionnaire offered at the end of the clinic, which they could send back at their convenience using a prepaid envelope. This questionnaire also assessed changes in their overall levels of concern about their medicines.

Patient self-reported adherence with individual secondary prevention medicines was re-evaluated 3-6 months post-clinic by asking patients to redo the relevant section of the MYMEDS questionnaire.

Prescription persistence with guidelines recommended secondary prevention medications was also assessed, at 11-12 months postdischarge, based on a sample of 50 patients managed by the consultant cardiology pharmacist within the medicines optimisation clinic. This was done remotely by reviewing their prescriptions and electronic records. Patients were given a persistence score using a locally developed tool (the 'Secondary Prevention Prescription Persistence Score' tool), based on the expected post-MI secondary prevention medications prescribed, the doses of beta blockers and ACE inhibitors (or ARBs) and eplerenone if ejection fraction $\leq 40 \%$. A score of $100 \%$ was given for patients prescribed all of the recommended medicines at the recommended doses. Patients scoring $<70 \%$ were deemed to be non-persistent. Rates of non-persistence were compared against a separate cohort of 50 post-MI patients (not needing further non-pharmacological interventions) going through the old service (ie, seen by a cardiologist outside the remit of the medicines optimisation clinic).

Finally, changes at system level following initiation of the medicines optimisation clinic were also evaluated. These included changes in mean patient waiting time from discharge to first outpatient cardiology review and changes in acute coronary syndrome (ACS)-related hospital readmission rates at 30,60 and 90 days after discharge after initiation of the service (1 October 2015 to 24 July 2016 (after this date, there were changes to the outpatient clinics that complicated comparison)) compared with a year before (1 October 2014 to 30 September 2015) as a baseline.

\section{Statistical analyses}

Descriptive statistics are provided. The baseline characteristics of patients who completed the questionnaire versus those who did not were compared using the independent samples t-test and $\chi^{2}$ test. The McNemar test was used to compare the proportion of patients receiving recommended doses of ACE inhibitors/ARBs, beta blockers, eplerenone and gastroprotective drugs, where indicated, before and after attending the clinic. Levels of patient concerns about their medicines before and after attending the clinic were assessed using the $\chi^{2}$ test. The $\chi^{2}$ test was also used to assess adherence with individual secondary prevention medicines before and after the clinic. For the prescription persistence data, two independent samples t-test was used to compare means and $\chi^{2}$ test to compare number of patients who attended the new and old service and had a score of $<70 \%$ using SPSS V.20. All reported $p$ values were two tailed.

\section{RESULTS \\ Demographics}

A total of 270 patients with MI who had completed their clinic experience before the cut-off date were included in the present analysis. Baseline patient characteristics are provided in table 1 . In total, $183(67.8 \%)$ were male, and the mean age was 67.3 years (range 33-95 years). Ninety-nine $(36.7 \%)$ had an ST-elevation MI (STEMI) and $171(63.3 \%)$ were patients with non-ST-elevation MI (NSTEMI). The majority had no history of prior MI $(\mathrm{n}=207 ; 76.7 \%)$.

Most patients $(\mathrm{n}=238 ; 88.1 \%)$ attended the standard rather than the advanced clinic. The mean time since discharge on attending the clinic was 49.4 days (range 9-195 days). Some patients were referred to the clinic rather than coming straight from discharge as per the normal pathway; if referred patients were excluded, the mean time to attending the clinic was 45.6 days (range 9-93 days).

Around a quarter of patients did not complete the questionnaire, either because they said that they did not receive it in the post or because they did not complete it and preferred instead to discuss the issues in person during the clinic. There were no significant differences in baseline characteristics between those who completed the questionnaire and those who did not, other than age (mean age $66.2 \pm 14.5$ years among non-respondents vs $70.5 \pm 10.9$ years among respondents; $\mathrm{p}=0.022$ ).

\section{Medicines optimisation}

Levels of medicines optimisation were improved substantially among patients attending the clinic. The proportion of patients receiving a recommended dose of an ACE inhibitor or ARB (where tolerated and not already on maximum dose at discharge) increased from $16.3 \%$ $(\mathrm{n}=33 / 203)$ to $73.9 \% \quad(\mathrm{n}=150 / 203) \quad$ (all uptitrated) $(\mathrm{p}<0.001)$. The remaining $26.1 \%$ of patients $(\mathrm{n}=53 / 203)$ who were on an ACE inhibitor or ARB could not be 


\begin{tabular}{|c|c|}
\hline Variable & $\begin{array}{l}\text { Baseline value } \\
(\mathrm{N}=270)\end{array}$ \\
\hline \multicolumn{2}{|l|}{ Sex, $n(\%)$} \\
\hline Male & $183(67.8)$ \\
\hline Female & $86(31.9)$ \\
\hline Not recorded & $1(0.4)$ \\
\hline Age (years), mean (range) & $67.3(33-95)$ \\
\hline \multicolumn{2}{|l|}{ Type of Ml, n (\%) } \\
\hline STEMI & $99(36.7)$ \\
\hline NSTEMI & $171(63.3)$ \\
\hline \multicolumn{2}{|l|}{ History of prior MI, n (\%) } \\
\hline Yes & $35(13.0)$ \\
\hline No & $207(76.7)$ \\
\hline Not recorded & $28(10.4)$ \\
\hline \multicolumn{2}{|l|}{ Comorbidities, n (\%) } \\
\hline Diabetes (any type) & $57(21.1)$ \\
\hline Hypercholesterolaemia & $40(14.8)$ \\
\hline Hypertension & $99(36.7)$ \\
\hline Left ventricular systolic dysfunction & $9(3.3)$ \\
\hline Chronic obstructive pulmonary disease & $24(8.9)$ \\
\hline Time since discharge (days), mean (range) & $49.4(9-195)$ \\
\hline \multicolumn{2}{|l|}{ Type of intervention, $\mathrm{n}(\%)$} \\
\hline Medical management & $76(28.1)$ \\
\hline Percutaneous coronary intervention & $194(71.9)$ \\
\hline \multicolumn{2}{|l|}{ Type of clinic at first visit, $n(\%)$} \\
\hline Standard & $238(88.1)$ \\
\hline Advanced & $32(11.9)$ \\
\hline
\end{tabular}

MI, myocardial infarction; NSTEMI, non-ST-elevation myocardial infarction; STEMI, ST-elevation myocardial infarction.

uptitrated (eg, due to hypotension or poor renal function) or needed their dose to be downtitrated (eg, due to hypotension or dizziness).

The proportion of patients receiving a recommended beta blocker dose (where tolerated and not already on maximum dose at discharge) increased from $6.2 \%$ $(\mathrm{n}=15 / 241)$ to $46.1 \% \quad(\mathrm{n}=111 / 241) \quad$ (all uptitrated) $(\mathrm{p}<0.001)$. The remaining $53.9 \% \quad(\mathrm{n}=130 / 241)$ either could not tolerate any further uptitration or uptitration could not be made in clinic (eg, due to an ACE inhibitor being uptitrated at the same time).

Antianginal drugs were optimised in $17.0 \%$ of patients $(n=46 / 270)$. Similarly, $20.8 \%$ of patients $(n=54 / 259)$ had their statin dose optimised (initiated a high-intensity statin, challenged non-tolerance or uptitrated in line with guidelines) and $14.1 \%(n=37 / 262)$ had their second antiplatelet dose optimised (switched due to side effects, or changed in line with triple therapy guidelines). Eplerenone and gastric protection were initiated in $82.4 \%$ $(n=14 / 17)$ and $25.0 \%(n=15 / 60)$ of patients, respectively, in whom they were indicated and who were not already receiving them. Hence, the number of patients who were suitable and initiated on eplerenone after
Table 2 Key changes in patient concerns before and up to a month after attending the medicines optimisation clinic (MYMEDS)

\begin{tabular}{|c|c|c|c|}
\hline Issue identified & Before clinic & After clinic & $P$ value \\
\hline $\begin{array}{l}\text { Patient understands the } \\
\text { reason for their cardiac } \\
\text { medicines }\end{array}$ & $143 / 196$ (73.0) & 192/194 (99.0) & $<0.001$ \\
\hline $\begin{array}{l}\text { Patient concern that cardiac } \\
\text { medicines will do more } \\
\text { harm than good }\end{array}$ & 62/187 (33.2) & 6/190 (3.2) & $<0.001$ \\
\hline $\begin{array}{l}\text { Patient sometimes forgets to } \\
\text { take medicines }\end{array}$ & $39 / 193(20.2)$ & $6 / 172(3.5)$ & $<0.001$ \\
\hline
\end{tabular}

Data are $\mathrm{n}(\%)$.

MYMEDS, My Experience of Taking Medicines questionnaire.

attending the clinic was $93.0 \% \quad(n=40 / 43)$ compared with $60.5 \%(\mathrm{n}=26 / 43)$ before the clinic $(\mathrm{p}<0.001)$. Similarly, the number of patients who were prescribed gastric protection, where indicated, increased from $65.3 \%$ $(\mathrm{n}=113 / 173)$ pre-clinic to $74.0 \%(\mathrm{n}=128 / 173)$ after the clinic $(\mathrm{p}<0.001)$.

\section{Patient concerns about secondary prevention}

Among patients who completed the MYMEDS questionnaire before attending the medicines optimisation clinic $(\mathrm{N}=204), 73.0 \%(\mathrm{n}=143 / 196)$ of those who answered this section said that they understood the reasons for their cardiac medicines (table 2). However, $33.2 \%(n=62 / 187)$ of those who answered this section expressed concern that their cardiac medicines would do more harm than good. After attending the clinic, feedback questionnaire responses (returned within a month post-clinic) showed that almost all patients felt that they now understood the reasons for their cardiac medicines (99.0\%; $\mathrm{n}=192 / 194)$ and few continued to believe that the medicines would do more harm than good $(3.2 \% ; n=6 / 190) \quad(\mathrm{p}<0.001$ compared with before the clinic). The proportion of patients who said that they sometimes forget to take their medicine also decreased from $20.2 \%(n=39 / 193)$ before the clinic to $3.5 \%(\mathrm{n}=6 / 172)$ afterwards $(\mathrm{p}<0.001)$.

\section{Patient experience of the service}

After attendance, patients were asked to rate their experience of the medicines optimisation clinic. Among 190 individuals who responded, the service was given a mean of score of 9.5 on a scale of 0 (very poor) to 10 (excellent). Furthermore, $77.6 \%$ ( $\mathrm{n}=152 / 196)$ strongly agreed and $22.4 \%(n=44 / 196)$ agreed that, overall, the clinic was valuable and they would recommend it for patients with heart disease (table 3). No patients disagreed.

When questioned about individual aspects of their participation in the clinic, such as having their questions answered and being involved in decision-making, $100 \%$ of patients either agreed or strongly agreed that it had been a positive experience (table 3 ). 
Table 3 Patient's experience of the medicines optimisation clinic

\begin{tabular}{|c|c|c|c|c|}
\hline & $\begin{array}{l}\text { Strongly } \\
\text { disagree }\end{array}$ & Disagree & Agree & $\begin{array}{l}\text { Strongly } \\
\text { agree }\end{array}$ \\
\hline I felt that I was listened to $(\mathrm{N}=200)$ & 0 & 0 & $47(23.5)$ & $153(76.5)$ \\
\hline All questions and concerns about my medicines were answered ( $\mathrm{N}=202)$ & 0 & 0 & $55(27.2)$ & $147(72.8)$ \\
\hline I felt reassured by the consultant cardiology pharmacist $(\mathrm{N}=201)$ & 0 & 0 & $43(21.4)$ & $158(78.6)$ \\
\hline I felt involved in the discussion and any decisions made were in agreement with me ( $N=201)$ & 0 & 0 & $47(23.4)$ & $154(76.6)$ \\
\hline This was a valuable clinic that I would recommend for patients with heart disease $(\mathrm{N}=196)$ & 0 & 0 & $44(22.4)$ & $152(77.6)$ \\
\hline
\end{tabular}

Data are $n(\%)$.

\section{Non-adherence and prescription non-persistence}

When attending the medicines optimisation clinic, rates of patient-reported non-adherence with individual secondary prevention medicines ranged between 13.6\% and $21.5 \%$ for different drug classes (table 4). When assessed again at 3-6 months after the clinic, rates of non-adherence had fallen to $4.2 \%-7.8 \%$. This equates to relative reductions in rates of non-adherence of $42.6 \%-$ $70.8 \%$ for the various classes of drugs. All of these reductions were statistically significant, apart from that relating to aspirin.

Prescription persistence with secondary prevention medications was assessed at 11-12 months post-MI discharge in a sample of 50 consecutive patients managed by the consultant cardiology pharmacist under the auspices of the medicines optimisation clinic. Results were compared against persistence among 50 consecutive patients treated under cardiologist care outside of the medicines optimisation clinic. Mean prescription persistence scores (SD, range) were $83.1 \%(9,64-100)$ and $76.5 \%(12,43-100)$, respectively $(\mathrm{p}=0.002)$. Rates of non-persistence (ie, a persistence score $<70 \%$ ) were $10.0 \%(\mathrm{n}=5)$ and $34.0 \%(\mathrm{n}=17)$, respectively, in patients attending or not attending the medicines optimisation clinic $(\mathrm{p}=0.004)$.

\section{Waiting times}

The medicines optimisation clinic led to a $44 \%$ reduction in mean waiting times from discharge to first outpatient cardiology review, from 88 days before the service to 49 days once the service began. Furthermore, the majority of patients were seen only by the consultant cardiology pharmacist, with fewer than 5\% requiring input from a cardiologist.

\section{Readmission rates}

The service was associated with a decrease in readmission rates. Compared with the previous year as a baseline, the proportion of patients readmitted to hospital with ACS within 30, 60 and 90 days of discharge was reduced by $42.9 \%$ (from $7 \%$ to $4 \%$ ), $50.0 \%$ (from $10 \%$ to $5 \%$ ) and $54.6 \%$ (from $11 \%$ to $5 \%$ ), respectively, after the service began operating.

\section{DISCUSSION}

The present study showed the positive impact of a novel post-MI clinic, which provided access to a consultant cardiology pharmacist or a cardiologist or both, on a range of measures, including optimisation of secondary prevention medicines and patient adherence. It demonstrates that collaboration and multidisciplinary working are central to the delivery of effective medicines optimisation. However, although patients had access to both a consultant cardiology pharmacist and a cardiologist, as needed, the majority of patients $(>95 \%)$ were seen only by the consultant cardiology pharmacist. Hence, this new service freed-up cardiology outpatient clinics and created more outpatient capacity. Importantly, patients were happy with the arrangement despite not being seen by the cardiologist; on the contrary, most were pleased with the pharmacist consultation and with the service in general as it adopted a patient-centred approach.

\begin{tabular}{|c|c|c|c|c|}
\hline $\begin{array}{l}\text { Secondary prevention } \\
\text { medicine }\end{array}$ & $\begin{array}{l}\text { Non-adherence rate } \\
\text { prior to clinic }\end{array}$ & $\begin{array}{l}\text { Non-adherence rate } 3-6 \\
\text { months post-clinic }\end{array}$ & $\begin{array}{l}\text { Relative reduction in } \\
\text { non-adherence }\end{array}$ & $P$ value \\
\hline ACE inhibitor /ARB & $27 / 178(15.2 \%)$ & $10 / 130(7.7 \%)$ & $49.3 \%$ & 0.046 \\
\hline Beta blocker & $23 / 169(13.6 \%)$ & $5 / 119(4.2 \%)$ & $69.1 \%$ & 0.008 \\
\hline Statin & $39 / 181(21.5 \%)$ & $8 / 127(6.3 \%)$ & $70.8 \%$ & $<0.001$ \\
\hline Clopidogrel/prasugrel/ticagrelor & $32 / 173(18.5 \%)$ & $7 / 126(5.6 \%)$ & $69.7 \%$ & 0.001 \\
\hline Aspirin & $24 / 176(13.6 \%)$ & $10 / 128(7.8 \%)$ & $42.6 \%$ & 0.112 \\
\hline
\end{tabular}

ARB, angiotensin receptor blocker.ACE, Angiotensin-Converting Enzyme. 
The mean time from discharge to clinic attendance was around 7 weeks (49.4 days), which was substantially lower than the mean waiting time to first outpatient cardiology review before the service began ( 88 days). One of the key early learnings of the project was the need to improve the outpatient booking system, and once that had been improved, patients were seen even more rapidly.

The model aligns with UK guidance from the National Institute for Health and Care Excellence (NICE), which recommends that structured medicines reviews should be considered for all patients with long-term conditions and/ or taking multiple medicines. ${ }^{10}$ This guidance also notes that organisations should decide locally on the most appropriate healthcare professional to lead these reviews, within the context of an MDT, and that a pharmacist may be an appropriate choice. ${ }^{10}$ The recent Carter review of productivity and performance in English NHS acute hospitals proposed that local trusts should deploy more clinical pharmacists, including pharmacist prescribers, to drive value in medicines spending, including optimisation activities. ${ }^{11}$

From an international perspective, a pharmacist-led model of medicines optimisation may not be applicable in all countries, particularly those where non-physician prescribing is not possible or where the role of the clinical pharmacist is less developed. However, structured reviews led by a physician rather than a pharmacist can be undertaken post-MI in most developed healthcare systems, and this work demonstrates their potential value.

The model adopted in this study aligned with the principles of medicines optimisation and adherence defined by NICE. ${ }^{510}$ In particular, the MYMEDS questionnaire enabled patients to think about actual or potential issues that might affect their ability to take their medicines and to derive maximum benefit from them. Furthermore, the consultation embraced a patient-centred approach and focused on the concerns that they highlighted in the MYMEDS questionnaire. Patients were encouraged to come primed with all of their questions and felt that the clinic was particularly fulfilling because it addressed their needs. They also felt more involved. Overall, this approach brought to reality the concept of 'shared decision-making', which is frequently recommended ${ }^{3510}$ but often difficult to deliver.

As a result, not only were secondary prevention medicines optimised but also substantial improvements in adherence were also achieved. This may have resulted from increased patient understanding of their medicines and the creation of specific action plans for overcoming barriers to adherence.

The association between improved adherence and improved long-term outcomes is well established. ${ }^{7} 812$ However, a recent Cochrane review of 182 trials of interventions designed to improve adherence to prescribed medications highlighted a lack of techniques with substantial impact on both adherence and outcomes. ${ }^{13}$ It is therefore encouraging that the present study was associated not only with an improvement in adherence but also improved outcomes, in the form of reduced hospital readmission rates at 30,60 and 90 days postdischarge. Although the mean time between discharge and attending the medicines optimisation clinic was 49 days across the data set, $22 \%$ of patients were seen within 30 days.

We must acknowledge some important limitations of the present study, in particular its retrospective design and the lack of a comparator arm for most outcome measures. A prospective, randomised controlled trial would certainly be valuable. In addition, self-reported adherence has its limitations, particularly with respect to overestimation. Adherence behaviour changes over time, and hence it would be useful to remeasure over longer-term time points. Another limitation that should be acknowledged is that the findings only apply to patients who did not need any further non-pharmacological coronary interventions post discharge. Not all patients completed the questionnaire and those who did not tended to be younger. Finally, there was a statistical analysis limitation resulting from the design of the study. While more powerful statistics could have been performed on paired data, not all of data could be paired because patient anonymity was offered in some questionnaires to reduce the risk of social desirability. Despite this, suitable statistical analyses were applied.

However, overall, the current study demonstrates that a medicines optimisation and patient adherence strategy based on a primarily pharmacist-led clinic supported by a cardiologist can improve both adherence and outcomes post-MI.

Acknowledgements We thank Dr Timothy Ryder from Biological Communications Limited for editorial assistance in drafting the manuscript. The service was paid for by Leeds Teaching Hospitals NHS Trust.

Contributors The project was led by RK with the support of the steering committee, which included all of the co-authors. The clinics were conducted in parallel with AH and JML. Data were collected with the help of NP. All planning and findings were presented to and discussed by the authors. The manuscript was drafted by the lead author and reviewed and adjusted based on suggestions from all of the co-authors. All authors gave final approval and agreed to be accountable for all aspects of the work. All revision were made by the lead author and coauthors reviewed and commented were appropriate.

Funding Funding for the proof-of-concept phase of the service was partly funded by Astra Zeneca, within a joint working agreement. The success of this phase led to the service to be fully commissioned by the Leeds Clinical Commissioning Groups and became the new standard service offered for our patients with myocardial infarction.

Competing interests None declared.

Patient consent for publication Not required.

Provenance and peer review Not commissioned; externally peer reviewed.

Data sharing statement The full data can be obtained by contacting the corresponding author.

Open access This is an open access article distributed in accordance with the Creative Commons Attribution Non Commercial (CC BY-NC 4.0) license, which permits others to distribute, remix, adapt, build upon this work non-commercially, and license their derivative works on different terms, provided the original work is properly cited, appropriate credit is given, any changes made indicated, and the use is non-commercial. See: http://creativecommons.org/licenses/by-nc/4.0

\section{REFERENCES}

1. National Institute for Health and Care Excellence. Myocardial infarction: cardiac rehabilitation and prevention of further cardiovascular disease. Clinical Guideline 172, 2013.

2. American College of Emergency Physicians, Society for Cardiovascular Angiography and Interventions, O'Gara PT, et al. 2013 ACCF/AHA guideline for the management of ST-elevation 
myocardial infarction: a report of the American College of Cardiology Foundation/American Heart Association Task Force on Practice Guidelines. J Am Coll Cardiol 2013;61:e78-140.

3. Ibanez B, James S, Agewall S, et al. 2017 ESC Guidelines for the management of acute myocardial infarction in patients presenting with ST-segment elevation: The Task Force for the management of acute myocardial infarction in patients presenting with ST-segment elevation of the European Society of Cardiology (ESC). Eur Heart $J$ 2018;39:119-77.

4. Rathod KS, Siddiqui S, Sin B, et al. Secondary prevention regimens and risk factors are not optimised in patients re-admitted with ACS. Br J Cardiol 2012:19:167-9.

5. National Institute for Health and Care Excellence. Medicines adherence: involving patients in decisions about prescribed medicines and supporting adherence. Clinical Guideline 76, 2009.

6. Naderi SH, Bestwick JP, Wald DS. Adherence to drugs that prevent cardiovascular disease: meta-analysis on 376,162 patients. Am J Med 2012;125:882-7.

7. Pittman DG, Chen W, Bowlin SJ, et al. Adherence to statins, subsequent healthcare costs, and cardiovascular hospitalizations. Am J Cardiol 2011;107:1662-6.
8. Choudhry NK, Glynn RJ, Avorn J, et al. Untangling the relationship between medication adherence and post-myocardial infarction outcomes: medication adherence and clinical outcomes. Am Heart $J$ 2014:167:51-8

9. Khatib R, Hall A. Adherence to secondary prevention medicines by coronary artery disease patients - exploring barriers to inform interventions. J Am Coll Cardiol 2012;59:E1460.

10. National Institute for Health and Care Excellence. Medicines optimisation: the safe and effective use of medicines to enable the best possible outcomes. NG5, 2015.

11. Lord Carter of Coles. Operational productivity and performance in English NHS acute hospitals: unwarranted variations. An independent report for the Department of Health by Lord Carter of Coles, 2016.

12. Chowdhury R, Khan H, Heydon E, et al. Adherence to cardiovascular therapy: a meta-analysis of prevalence and clinical consequences. Eur Heart J 2013;34:2940-8.

13. Nieuwlaat R, Wilczynski N, Navarro T, et al. Interventions for enhancing medication adherence. Cochrane Database Syst Rev 2014;11:CD000011. 